ISSN: 2638-5279

Volume 1, Issue 2, 2018, PP: 50-54

\title{
Relation between Toxoplasma Infection and Schizophrenia in Yazd, Iran
}

\author{
Hossein Hadinedoushana, Jamshid Ayatollahi' ${ }^{\text {b* }}$, Mahmood Rafeeianc, Seyed Hossein Shahcheraghi ${ }^{\mathrm{b}}$ \\ aDepartment of Immunology, Reproductive Immunology Research Center, Shahid Sadoughi University of \\ Medical Sciences, Yazd, Iran. \\ ${ }^{b}$ Infectious Diseases Research Center, Shahid Sadoughi Hospital, Shahid Sadoughi University of Medical \\ Sciences, Yazd, Iran. \\ 'Psychologist, Shahid Sadoughi University of Medical Sciences, Yazd, Iran. \\ jamshidayatollahi@yahoo.com \\ *Corresponding Author: Dr. Jamshid Ayatollahi, Infectious Diseases Research Center, Shahid Sadoughi Hospital, \\ Shahid Sadoughi University of Medical Sciences, Yazd, Iran.
}

\section{Abstract}

Introduction: Schizophrenia is a serious neuropsychiatric disease of uncertain etiology. Epidemiological and neuropathological studies have also indicated that some cases of schizophrenia may be associated with infectious agents. Toxoplasma gondii (T.gondii) is a coccidian parasite found worldwide, that infects nearly one third of humanity. To investigate a potential association between Toxoplasma infection and schizophrenia.

Materials and Methods: This research was carried out as case control study on two different groups. Case group consisted of eighty-eight who were first hospitalized for schizophrenia. The control population consisted of ninety health blood donors referred to Yazd blood bank. We measured the levels of class-specific IgG, IgM, antibodies to T. gondii in the serum samples using ELISA method according to manufacture instruction.

Results: There was no significant difference in the age of two groups (38.4 \pm 12.8 vs. $39.5 \pm 13.5$ years). 46 out of 90 individual in control (51.1\%) had IgG antibody higher than $15 \mathrm{Iu} / \mathrm{ml}$ and considered protective to T. gondii infection. Also 44 out of 88 (50\%) in case group had IgG level higher than $15 \mathrm{Iu} / \mathrm{ml}$. There was no significant difference in IgG levels in two groups $(P=0.4)$. There was insignificant difference between IgG levels and age. Only 4 sera in control and 5 sera in case group were positive for anti-T.gondii from IgM class.

Conclusions: Our findings indicate that T.gondii infections may not play a role in the clinical manifestation of patients with schizophrenia. Additional studies will have to elucidate the causative relation between infection with T. gondii and psychiatric disorders.

Keywords: Schizophrenia, Toxoplasma gondii, Infection, Protective

\section{INTRODUCTION}

Schizophrenia is a tenacious mind infection that is generally first apparent in late immaturity or early adulthood and continues along a lifelong course [1]. If a bacterial and parasitic agent helps to schizophrenia pathogenesis, there are various natural and epidemiological prerequisites that the operator must satisfy to be a conceivable applicant agent [2]. For example, it may be normal that the applicantirresistible operator or gathering of specialists show an overall conveyance as is comparative for schizophrenia [3].
Moreover, given the schizophrenia is likely turmoil of neurodevelopment, the planning of presentation to a putative irresistible specialist is probably going to happen amid eras vital for neurodevelopment $[4,5]$.

Based on studies, several main agents, infectious factors among them, have been offered for schizophrenia. The intracellular parasite Toxoplasma gondii (T. gondii) is one of these irresistible specialists because of its relationship with cerebrum brokenness and its neurotropism [6].

Toxoplasmosis is the most frequent protozoa agent 
Relation between Toxoplasma Infection and Schizophrenia in Yazd, Iran

infecting one third of the global human population [7]. This intracellular parasite changes the expression of host cell genes (including brain cells) and holds on in the form of cysts. These cysts can reactivate and discharge organisms by neospread all through the host, based on his/her immune condition [8] $T$. gondii's neurotropism and its effect on dopamine have been proved [9] and dopamine unsettling influences might be related with insane or mood episodes. The effects of parasite is related to brain dysfunction [10].

Almost all previous studies concerning the relation of T.g. infection and schizophrenia were based on indirect measurement by investigating antibodies specially $\operatorname{IgG}[6,11]$, and the results were uncertain. Serological testing for toxoplasmosis relies on investigation of immunoglobulin $\mathrm{M}$ (IgM) and immunoglobulin $\mathrm{G}$ (IgG). A positive IgM test is usually a marker of an acute infection. However, IgM can persist for several times after an acute parasitic infection, therefore, making the distinction between an acute and a chronic infection challenging. High-IgG-avidity test results in patients with positive IgM test titers can establish that the patient has been infected for almost 3 months, but low-avidity test results alone are not necessarily diagnostic of an acute infection [12].

The findings would further help in understanding the etiology of schizophrenia and its relation with toxoplasma infection. Therefore, our study will provide a better understanding of defining

the possible association between Toxoplasma exposure and the risk of schizophrenia in the central area of Iran specially Yazd city.

\section{Materials and Methods}

\section{Study Population}

This case-control study which was conducted in Yazd, the capital of Yazd province, central area of Iran. Cases were 90 individuals with psychiatric diagnosis of schizophrenia based on Diagnostic and Statistical Manual of Mental Disorders, who were referring or hospitalized in the center for psychiatric disorders in Taft County from functions of Yazd. Control subjects were consenting, apparently healthy blood donors with no history of psychiatric illness or any other health problem, frequency-matched with patients. An easy census method was used to select these items.

\section{Serological Examination}

Blood samples were collected from the study population $(5 \mathrm{ml})$ and sera were stored at $-70{ }^{\circ} \mathrm{C}$ until use.

Forserologicalexamination and detection ofantibodies, commercial enzyme linked immunosorbent assay (ELISA) kits (Dia Plus, Canada) were used according to the instructions of the manufacturer. Samples with an absorbance value above the cut-off level (greater than or equal to $20 \mathrm{IU} / \mathrm{ml} \mathrm{OD}$ ) were considered positive about IgG. This value for IgM positive was greater than cut off OD value.

\section{Data Collection}

In both group, data about age, sex, education, marital status, contact with catand history of psychiatricillness or other major health problems were collected at the time of blood donation using a brief questionnaire.

In schizophrenia patients, we also noted duration of illness, number of hospitalizations, history

of suicide attempts, and history of smoking/drug from hospital records. History of suicide attempts were defined as explicit statements referring to attempted suicide in the patient's record. Duration of illness was defined based on the first occurrence of psychotic symptoms as stated in the patient's history. Age of onset of schizophrenia symptoms was calculated based on the subtraction of duration of illness from the current age.

\section{Statistical Analysis}

Statistical analysis was conducted using SPSS software (Version 16.0; SPSS, Inc., Chicago, USA). The frequency distribution of IgG and IgM antibodies in the two groups was statistically analyzed by Chisquare test. Comparison of mean of IgG antibody level in two groups was determined by t-test. Finally, the association between age and IgG antibody production was performed using t-test.

\section{RESULTS}

The age mean in the apparently healthy control group was $38.4 \pm 12.8$ years and in the group with schizophrenia was $39.5 \pm 13.5$ years. The minimum age in the control group was 18 and maximum 57 years, with a median of 35.5 years. The minimum age in the patient group was 20 and maximum 55 years, with a 
Relation between Toxoplasma Infection and Schizophrenia in Yazd, Iran

median of 38.5. There was no significant difference between the age mean of two groups $(\mathrm{P}=0.4)$.

In terms of education, $43(47.8 \%)$ were under the diploma, 26 (28.9\%) had diplomas and 18 (20\%) were bachelor and 3 (3.3\%) had master's degree and higher while in the schizophrenic group 58 (65.9\%) had subdiploma, 27 (30.7\%) had diplomas and 2 (2.3\%) were bachelor and only one person (1.1\%) had a master's degree or higher. The number of people with the subdiploma degrees in the patient group was much higher toward the control group and the number of people with undergraduate degrees in the apparently healthy group was much higher than the patient group.

$64(72.7 \%)$ of the patients were male and $24(27.3 \%)$ were female. In the healthy control group, 67 (74.4\%) were male and 23 (25.6\%) were female. There was no significant difference in gender between the two groups $(p=0.2)$. Four serum samples in the control group (three males and one female in the ages of 22,32, 27 , and 52 years old) and five serum samples in the patients group of schizophrenia (three males and two females aged 24, 32, 35, 39, 46 years old) contained
IgM antibody against Toxoplasma gondii, indicating a recent infection with this parasite.

In terms of the small number of positive cases in both groups, there was no possibility for statistical comparison between the two groups. 46 cases (51.1\%) of the control group had antibody against Toxoplasma gondii from class of IgG that was in excess of $20 \mathrm{IU} / \mathrm{ml}$, which was considered as positive antibody about IgG, indicating infection with this parasite in the period from life.

$44(48.9 \%)$ of the cases had an IgG level against the parasite less than $10 \mathrm{IU} / \mathrm{ml}$, which was considered negative in terms of IgG. In the patient group, 44 (50\%) of the patients had an IgG antibody level above 20 IU/ $\mathrm{ml}$, which was considered as positive. In 44 samples (50\%), the IgG antibody levels were less than $10 \mathrm{IU} /$ $\mathrm{ml}$, which was considered negative.

As shown in Table 1, the anti-Toxoplasma gondii antibody concentration (IgG class) was significantly higher in patients with acute schizophrenia than in control group $(\mathrm{P}=0.03)$.

Table 1. IgG rate against Toxoplasma in patients with acute schizophrenia and control

\begin{tabular}{|l|c|c|c|c|}
\hline \multicolumn{1}{|c|}{ IgG rate } & Mean \pm SD & Minimum & Maximum & P-value \\
\hline IgG rate in the control subjects & $69.8 \pm 105.9$ & 1.9 & 439.1 & 0.03 \\
\hline IgG rate in patients with acute schizophrenia & $82.9 \pm 114.3$ & 2.8 & 491 & 439.1 \\
\hline IgG rate in the positive samples of control group & $125.8 \pm 101.5$ & 33.6 & 4 & 0.04 \\
\hline IgG rate in the positive samples of patient group & $137.7 \pm 123.5$ & 21.9 & 491 & \\
\hline
\end{tabular}

The IgG mean in 36 males patients in the schizophrenic group considered as IgG positive was $142.3 \pm 132.8$ IU/ $\mathrm{ml}$, and in 13 positive women in this group was 133.6 $\pm 93.5 \mathrm{IU} / \mathrm{ml}$ that it was no statistically significant $(\mathrm{P}$ $=0.174$ ). Also, the difference of IgG levels mean in the control men and women who were considered positive for IgG was not significant $(\mathrm{P}=0.2)$.

The relationship between age and antibody level of IgG against $T$. gondii parasite in positive subjects in the group of patients with schizophrenia $(P=0.2, R$ $=0.07)$ ) as well as in those considered as positive for IgG in the control group was not observe $(\mathrm{P}=0.15, \mathrm{R}$ $=0.09$ ).

The correlation between IgG level in the control group, which were considered as positive for IgG and education level was no seen $(\mathrm{P}=0.2)$. Also, there was no significant correlation between IgG level in patients with positive antibody and education level $(\mathrm{P}=0.3)$.

\section{Discussion}

In our study, there was no significant difference between the age mean of two groups. Also, In terms of the small number of positive cases in both groups, there was no possibility for statistical comparison between the two groups. IgG antibody level against Toxoplasma was significantly higher in patients with acute schizophrenia than in control group. Only 4 sera in control and 5 sera in case group were positive for anti-T.gondii from IgM class. It proved that T. gondii infections may not play a key role in the clinical manifestation of patients with schizophrenia in the central area of Iran.

An examination intended to inspect the serofrequency 
Relation between Toxoplasma Infection and Schizophrenia in Yazd, Iran

and serointensity of Toxoplasma gondii ( $\mathrm{Tg}$ ) IgG, IgM, and DNA among cases with schizophrenia disease. Results demonstrated that IgM between patients with schizophrenia and controls had not significant difference $(P>0.005)$. Also, there was no significant difference in both serointensity of $\mathrm{Tg}$ IgG and DNA between cases with schizophrenia and control individuals [13].

Another study was performed in a Mexican population. Both the seroprevalence and the rate of T.g. IgG antibodies were higher in schizophrenic cases $(10 / 50 ; 20 \%)$ toward controls. The IgG levels higher than $150 \mathrm{IU} / \mathrm{ml}$ were more frequently seen in patient cases. One (50\%) of the two patients with as of late analyzed schizophrenia and none of the controls had T. gondii IgM [12].

Cetinkaya, et al investigated the seropositivity rate for anti-Toxoplasma antibodies by ELISA in patients with schizophrenia to discover a conceivable relationship between schizophrenia and $\mathrm{T}$. gondii. Their findings showed that the seropositivity level for anti-Toxoplasma IgG antibodies among schizophrenia patients $(66 \%)$ was significantly more than among patients with depressive issue or sound volunteers [14].

Hamidinejat $\mathrm{H}$, et al showed that the positivity rate of anti-T. gondii IgG antibodies among individuals with schizophrenia (57.1\%) was significantly higher than in healthy controls (29.2\%). There were no associations between immune status ratio values and the risk of schizophrenia. The weight of confirm approach utilizing the Bradford Hill criteria showed a $92 \%$ probability of a causal relation [15].

\section{CONCLUSION}

Our study indicated that T.gondii infections may not play a main and key role in the clinical manifestation of patients with schizophrenia.

\section{Acknowledgment}

The authors wish to thank the staff of Infectious Diseases Research Center of Shahid Sadoughi University of Medical Sciences, Yazd. Also, we thank Mrs. Fatemeh Shiri and Mrs. Mahshid Hosseini from students of lab sciences.

\section{REFERENCES}

[1] Brown AS, Derkits EJ. Prenatal infection and schizophrenia: a review of epidemiologic and translational studies. American Journal of Psychiatry. 2009;167(3):261-80.

[2] Brown AS, Patterson PH. Maternal infection and schizophrenia: implications for prevention. Schizophrenia bulletin. 2010;37(2):284-90.

[3] FlegrJ. Influence oflatent Toxoplasma infection on human personality, physiology and morphology: pros and cons of the Toxoplasma-human model in studying the manipulation hypothesis. Journal of experimental Biology. 2013;216(1):127-33.

[4] Fineberg AM, Ellman LM, Buka S, Yolken R, Cannon TD. Decreased birth weight in psychosis: influence of prenatal exposure to serologically determined influenza and hypoxia. Schizophrenia bulletin. 2012;39(5):1037-44.

[5] Severance EG, Gressitt KL, Buka SL, Cannon TD, Yolken RH. Maternal complement C1q and increased odds for psychosis in adult offspring. Schizophrenia research. 2014;159(1):14-9.

[6] Torrey EF, Bartko JJ, Lun Z-R, Yolken RH. Antibodies to Toxoplasma gondii in patients with schizophrenia: a meta-analysis. Schizophrenia bulletin. 2006;33(3):729-36.

[7] Kim K, Weiss LM. Toxoplasma: the next 100 years. Microbes and infection. 2008;10(9):978-84.

[8] Carruthers VB. Host cell invasion by the opportunistic pathogen Toxoplasma gondii. Acta tropica. 2002;81(2):111-22.

[9] Prandovszky E, Gaskell E, Martin H, Dubey J, Webster JP, McConkey GA. The neurotropic parasite Toxoplasma gondii increases dopamine metabolism. PloS one. 2011;6(9):e23866.

[10] Abi-Dargham A. Schizophrenia: overview and dopamine dysfunction. The Journal of clinical psychiatry. 2014;75(11):e31-e.

[11] Daryani A, Sharif M, Hosseini SH, Karimi SA, Gholami S. Serological survey of Toxoplasma gondii in schizophrenia patients referred to Psychiatric Hospital, Sari City, Iran. Trop Biomed. 2010;27(3):476-82.

[12] Alvarado-Esquivel C, Urbina-Álvarez JD, EstradaMartínez S, Torres-Castorena A, Molotla-de-León $\mathrm{G}$, Liesenfeld $\mathrm{O}$, et al. Toxoplasma gondii infection and schizophrenia: a case control study in a low 
Relation between Toxoplasma Infection and Schizophrenia in Yazd, Iran

Toxoplasma seroprevalence Mexican population. Parasitology international. 2011;60(2):151-5.

[13] Omar A, Bakar OC, Adam NF, Osman H, Osman A, Suleiman $\mathrm{AH}$, et al. Seropositivity and serointensity of Toxoplasma gondii antibodies and DNA among patients with schizophrenia. The Korean journal of parasitology. 2015;53(1):29.

[14] Cetinkaya Z, Yazar S, Gecici O, Namli MN. AntiToxoplasma gondii antibodies in patients with schizophrenia-preliminary findings in a Turkish sample. Schizophrenia bulletin. 2007;33(3):78991.

[15] Hamidinejat $H$, Ghorbanpoor $M$, Hosseini $H$, Alavi SM, Nabavi L, Jalali MHR, et al. Toxoplasma gondii infection in first-episode and inpatient individuals with schizophrenia. International journal of infectious diseases. 2010;14(11):e978e81.

Citation: Hossein Hadinedoushan, Jamshid Ayatollahi, Mahmood Rafeeian, Seyed Hossein Shahcheraghi. Relation between Toxoplasma Infection and Schizophrenia in Yazd, Iran. Open Access Journal of Internal Medicine. 2018; 1(2): 50-54.

Copyright: (C) 2018 Hossein Hadinedoushan, Jamshid Ayatollahi, Mahmood Rafeeian, Seyed Hossein Shahcheraghi. This is an open access article distributed under the Creative Commons Attribution License, which permits unrestricted use, distribution, and reproduction in any medium, provided the original work is properly cited. 